\title{
Separation of vanadium and molybdenum from aqueous solution using PEG2000 + sodium sulfate + water aqueous two-phase system
}

\author{
Yajing Pan ${ }^{1} \cdot$ Xiao Sun ${ }^{1} \cdot$ Yongqiang Zhang ${ }^{1}$
}

Received: 19 July 2019 / Accepted: 15 October 2019 / Published online: 24 October 2019

(c) Springer Nature Switzerland AG 2019

\begin{abstract}
The separation of vanadium (V) and molybdenum (VI) was studied by co-extraction with an aqueous two-phase system formed by PEG2000 + sodium sulfate + water and by selective stripping with another aqueous two-phase system formed by ammonium sulfate solution and PEG2000 phase loaded vanadium and molybdenum. The effect of aqueous $\mathrm{pH}$, concentration of vanadium and molybdenum, temperature on the co-extraction and concentration of ammonium sulfate, temperature and phase ratio on selective stripping separation of vanadium and molybdenum was investigated. The experimental results on co-extraction of vanadium and molybdenum indicated that the co-extraction rate of both metals is sensitive to aqueous $\mathrm{pH}$, and their extraction rate can achieve above $97.54 \%$ and $99.49 \%$, respectively, when the $\mathrm{pH}$ value of aqueous solution is at 2.0, the molar ratio of vanadium to molybdenum is about 1.5 , the concentration of PEG2000 is $20 \%$, and the temperature is $313.15 \mathrm{~K}$, respectively. The co-extraction rate of both metals decreases slightly with an increase in temperature from 313.15 to $343.15 \mathrm{~K}$. The extraction isotherm and McCabe-Thiele method showed that two theoretical stages are needed when concentration of vanadium and molybdenum decreases from initial $12 \mathrm{~g} / \mathrm{L}$ and $10 \mathrm{~g} / \mathrm{L}$ to $0.1 \mathrm{~g} / \mathrm{L}$ under the optimal conditions. The results on selective stripping of both metals from loaded PEG2000 phase to aqueous phase indicated that the stripping rate and precipitation rate of vanadium are evidently impacted by aqueous $\mathrm{pH}$ within 8.0 11.0 and then a little influenced by other parameters. The stripping rate of molybdenum is relatively lower than that of vanadium and is influenced by all kinds of stripping conditions. The stripping rate and precipitation rate of vanadium achieve $99.88 \%$ and $98.84 \%$, respectively, and stripping rate of molybdenum achieves $81.99 \%$ under the optimal stripping conditions of $\mathrm{O} / A=2: 1$, stripping temperature $343.15 \mathrm{~K},\left(\mathrm{NH}_{4}\right)_{2} \mathrm{SO}_{4}(w / w) 40 \%$ and the $\mathrm{pH}$ of aqueous solution at 10.0 .
\end{abstract}

Keywords Aqueous two-phase system · Separation · Polyethylene glycol · Sodium sulfate · Vanadium · Molybdenum

\section{Introduction}

Vanadium and molybdenum are widely used in aerospace, electronic information, industrial production and many other technical fields for their unique properties of corrosion resistance, high temperature resistance and wear resistance, which have greatly stimulated the demanding in recent years. At the same time, it is urgent to develop and reuse its secondary resources as the world's resources continue to be mined and high-grade ore is increasingly depleted. Among the secondary resources, waste catalysts are undoubtedly more important. The waste hydrodesulfurization catalysts replaced in petroleum refineries contain higher precious metals than precious metal ores. They are considered as an important source of strategic metals such as vanadium, molybdenum, nickel and cobalt $[1,2]$. The reasonable recovery of spent catalysts not only has great economic benefits, but also solves the

$\triangle$ Yongqiang Zhang, 13784195390@163.com | ${ }^{1}$ Hebei Key Laboratory of Applied Chemistry, School of Environmental and Chemical Engineering, Yanshan University, Qinhuangdao 066004, China. 
environmental pollution problems associated with their disposal [3]. At present, there are many reports on the recovery of metal vanadium and molybdenum from spent catalysts [4-9].

In recent decades, many researchers have studied and developed methods for separating and recovering vanadium and molybdenum from leaching solutions. The main methods for the separation of vanadium and molybdenum are ion exchange [10, 11], solvent extraction [12-14] and chemical precipitation [15]. Among them, the solvent extraction method is the most widely used depending on its simple operation, selective control of separation and complete separation. Some researchers use different extractants to extract and separate vanadium and molybdenum, such as Alamine-336 [16], EHEHPA [17], LIX 63 [18] and Cyphos IL.

However, most organic solvents are toxic, flammable and volatile. Using of organic solvents is very harmful to human health and the environment and does not conform to the concept of green chemistry [19]. In addition, although the ion exchange method can effectively separate metals and obtain high-quality products, the ability to load metals is limited, and it cannot meet the scale production of the factory, which leads to the application limited. Therefore, it is necessary to develop a green and environmentally friendly method to separate vanadium and molybdenum. On this basis, we found that the aqueous two-phase system can effectively extract and separate vanadium and molybdenum. Moreover, up to now, it is noteworthy that there is no report on the separation of vanadium and molybdenum by aqueous two-phase system. Compared with the traditional separation methods mentioned above, this method not only balances or even exceeds its efficiency, but also satisfies the outstanding advantages of energy saving, simplicity and time saving. It is worth mentioning that the organic solvents are absent, which actively responds to the safety concept of "green chemistry" for aqueous two-phase extraction systems.

Aqueous two-phase systems has become more and more attractive in recent years due to its mild operating conditions, scale-up easily and no pollution to the environment. It is a promising new separation technology [20]. At present, many researchers have successfully applied this technology to bioengineering [21, 22], fermentation engineering [23], separation of organic matter [24] and other fields. There are also many studies on the application of this technology to extraction and separation of metal ions, for example the separation of $\mathrm{Hg}(\mathrm{II}), \mathrm{Zn}(\mathrm{II})$ and $\mathrm{Co}(\mathrm{II})$ by Dehghani [25], the separation of $\mathrm{Ca}$ (II), $\mathrm{Mg}$ (II) and $\mathrm{Ni}$ (II) by Rodrigues [26] and the extraction of $\mathrm{Bi}$ (III) subsalicylate by Sen [27]. The extraction and separation performance and the extraction mechanism of molybdenum and tungsten with the aqueous twophase system were reported by our early research work [28, 29], and we also used the same system to investigate the extraction and separation of vanadium. It was found that for the extraction of alone vanadium and molybdenum, molybdenum is more easily extracted into the PEG-rich phase, while vanadium is difficult to extract into the PEG-rich phase without the addition of a charge modifier under the acidic conditions [30]. In addition, the conclusions of the partition of Molybdate ion in aqueous two-phase system formed by $\mathrm{CuSO}_{4}+\mathrm{PEG} 4000+\mathrm{H}_{2} \mathrm{O}$ were obtained by Duran [31].

In this work, the purpose is to investigate the co-extraction and separation performance of vanadium $(\mathrm{V})$ and molybdenum( $\mathrm{VI}$ ) with aqueous two-phase system composed of PEG $2000+\mathrm{Na}_{2} \mathrm{SO}_{4}+\mathrm{H}_{2} \mathrm{O}$ without any extractant on the basis of our previous research work. By a series of basic experimental studies, we found that $\mathrm{pH}$, concentration and $T$ have great influence on the experimental results, while other factors (such as stirring rate and aging time) have little or no influence, so we will not give a single narrative here. The article mainly narrates the effect of aqueous $\mathrm{pH}$, initial vanadium and molybdenum concentration, temperature on co-extraction of vanadium and molybdenum, concentration of ammonium sulfate and ammonium hydroxide and temperature on selective stripping separation of vanadium and molybdenum.

\section{Experiments}

\subsection{Reagents}

PEG2000 with an average molar mass $1900 \sim 2200 \mathrm{~g} / \mathrm{mol}$ was obtained from Xilong Chemical Co. Ltd. (GuangDong, China). The stock solutions of molybdenum and vanadium were prepared by dissolving $\mathrm{Na}_{2} \mathrm{MoO}_{4}$ and $\mathrm{NaVO}_{3}$ in distilled water, respectively. Sodium sulfate, ammonium sulfate, sodium metavanadate and sodium molybdate were obtained from West Long Science Co. Ltd. (GuangDong, China), while sulfuric acid was supplied by Tianjin Kermel Chemical Reagent Co. Ltd. (Tianjin, China). All reagents were analytical grade with the purity of above $99 \%$ and directly used. Distilled water was used in all experiments.

\subsection{Experimental procedure}

\subsubsection{Extraction}

The aqueous two-phase system was prepared with PEG2000, sodium sulfate stock solution and vanadium and molybdenum stock solutions, and the system was composed of $20 \%(w / w)$ PEG2000, $10 \%(w / w)$ sodium sulfate, $0.2 \%(w / w)$ vanadium and $0.5 \%(w / w)$ molybdenum. The $\mathrm{pH}$ of mixture solutions was adjusted to 1.5, 2.0, 3.0, 4.0 and 5.0, respectively, by acidometer (PHS-3C, Shanghai, China) with $2 \mathrm{~mol} / \mathrm{L}$ sulfuric acid. The mixture was stirred 
vigorously at $313.15 \mathrm{~K}$ for $30 \mathrm{~min}$ in a thermostatic magnetic stirrer for fully blending and then placed in a constant temperature water bath at the same temperature for $24 \mathrm{~h}$. All aqueous two-phase extraction systems were placed in $50-\mathrm{mL}$ glass graduated tubes for phase separation. After the phase separation was finished, the volume of the upper phase (extract phase) and lower phase (saltrich phase) was accurately recorded for calculating the volume ratio. The molybdenum and vanadium in salt-rich phase were appropriately diluted with distilled water and analyzed by inductively couple plasma atomic emission spectrometry (ICP-AES 6300 Radial, Thermo Scientific, USA). Parallel experiments were carried out during the experiments to ensure the accuracy of the data.

The molybdenum and vanadium concentration in PEGrich phase was determined with material balance method based on phase volume [Eq. (1)]. The phase volume ratio, partitioning coefficient and extraction rate of molybdenum and vanadium were calculated according to Eqs. (2), (3) and (4).

$c_{\mathrm{At}}=\frac{m-V_{\mathrm{b}} c_{\mathrm{Ab}}}{V_{\mathrm{t}}}$

$R=\frac{V_{\mathrm{t}}}{V_{\mathrm{B}}}$

$D=\frac{c_{\mathrm{At}}}{c_{\mathrm{Ab}}}$

$E(\%)=\frac{R D}{R D+1} \times 100 \%$

where $c_{\mathrm{At}}$ and $c_{\mathrm{Ab}}$ are vanadium/molybdenum concentration in PEG-rich phase and salt-rich phase $(\mathrm{g} / \mathrm{L})$, respectively, $V_{\mathrm{b}}$ and $V_{\mathrm{t}}$ represent the volume in salt-rich phase and PEG-rich phase (L), respectively, $R$ is phase volume ratio (PEG-rich phase volume to salt-rich phase volume), $D$ is partitioning coefficient of vanadium/molybdenum, $E$ is extraction rate percentage of vanadium/molybdenum and $m$ is the mass of vanadium/molybdenum added ( $\mathrm{g}$ ).

\subsubsection{Stripping}

A certain mass of PEG2000 phase loaded vanadium and molybdenum and a certain amount of $\left(\mathrm{NH}_{4}\right)_{2} \mathrm{SO}_{4}$ solution were placed in a $50-\mathrm{mL}$ beaker, the mixture was adjusted to a certain $\mathrm{pH}$ with ammonium hydroxide, and finally, a certain amount of distilled water was added. At the temperature of $323.15-363.15 \mathrm{~K}$, the mixed solutions were stirred with a thermostatic magnetic stirrer for 40-120 min, and then, the phase separation was completed at room temperature. The volumes of the upper and lower phases and the precipitate were accurately recorded. The vanadium and molybdenum in the salt-rich phase and the PEGrich phase were diluted with distilled water for appropriate multiples and then were analyzed by inductively coupled plasma atomic emission spectrometry. The stripping percentage was calculated by Eq. 5 .

$E_{\text {strip }}=\frac{V_{\text {bs }} c_{\text {Abs }}}{m_{0}} \times 100 \%$

where $E_{\text {strip }}$ is the stripping percentage (\%), $V_{\mathrm{bs}}$ is the volume in stripping salt-rich phase (L), $c_{\mathrm{Abs}}$ are the vanadium/ molybdenum concentration in stripping salt-rich phase $(\mathrm{g} / \mathrm{L})$ and $m_{0}$ are the vanadium/molybdenum mass in loaded extraction phase $(\mathrm{g})$.

\section{Results and discussion}

\subsection{Co-extraction of vanadium and molybdenum}

\subsubsection{Effect of aqueous $\mathrm{pH}$}

The effect of aqueous $\mathrm{pH}$ varying from 1.5 to 5.0 on extraction of vanadium and molybdenum is shown in Fig. 1. It can be seen from the figure that the extraction rate of vanadium goes up evidently maximum $95.23 \%$ at $\mathrm{pH}=2.0-3.0$, and the extraction rate of molybdenum remains the higher value above $99.48 \%$ at $\mathrm{pH}=1.5-3.0$, and this is due to forming heteropolyacid anions of vanadium and molybdenum, such as $\mathrm{HMo}_{4} \mathrm{~V}_{2} \mathrm{O}_{19}{ }^{3-}, \mathrm{Mo}_{5} \mathrm{VO}_{19}{ }^{3-}$, $\mathrm{Mo}_{7} \mathrm{VO}_{26}{ }^{5-}, \mathrm{HMoV}_{9} \mathrm{O}_{28}{ }^{4-}, \mathrm{Mo}_{2} \mathrm{~V}_{8} \mathrm{O}_{28}{ }^{4-}$ and $\mathrm{HMoV}_{9} \mathrm{O}_{28}{ }^{4-}$, in aqueous media containing $\mathrm{Mo}(\mathrm{VI})-\mathrm{V}(\mathrm{V})-\mathrm{H}_{2} \mathrm{O}$ system according to Liao's report [32]. These heteropolyacid anions have relatively high hydrophobicity and also increase

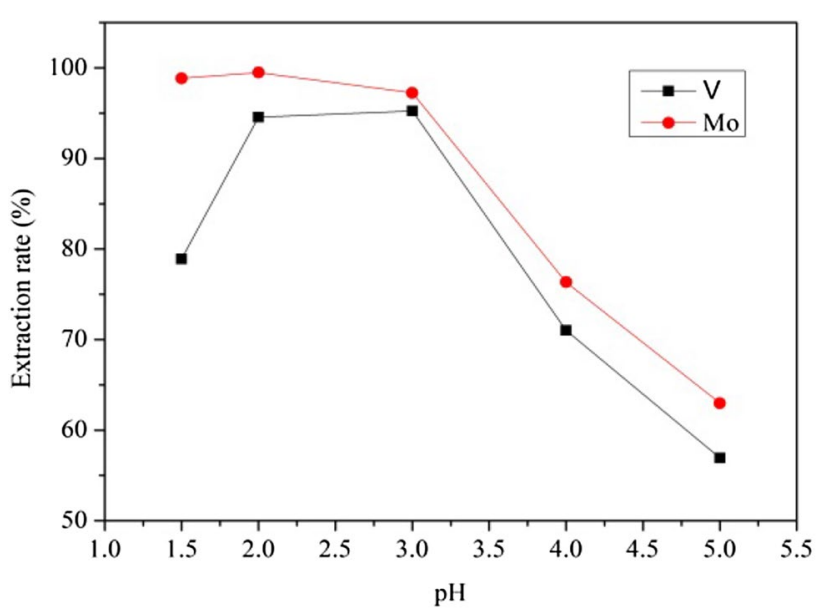

Fig. 1 Effect of aqueous $\mathrm{pH}$ on the co-extraction of vanadium and molybdenum 
mutual solubility with PEG2000-rich phase having hydrophobic environment, in the meanwhile, the electrostatic attraction generated between heteropolyacid anion and quasi-cation of $\mathrm{C}-\mathrm{O}-\mathrm{C}$ or $-\mathrm{OH}$ protonated in PEG2000 molecule promotes the extraction rate of heteropolyacid anion. The subsequent studies were conducted at $\mathrm{pH}$ of 2.0. The extraction rates of vanadium drop rapidly when the $\mathrm{pH}$ value is less than 2.0 , it can be explained that $\mathrm{VO}^{2+}$ is formed, and the electrostatic repulsion is produced between protonated $\mathrm{C}-\mathrm{O}-\mathrm{C}$ or $-\mathrm{OH}$ in PEG2000 molecule and $\mathrm{VO}^{2+}$, resulting in lower extraction rate of vanadium. The extraction rate of vanadium and molybdenum decreases when the $\mathrm{pH}$ value is more than 3.0, showing that the isopolyacid anion of vanadium and molybdenum gradually increases and their patterns are various, such as $\mathrm{HV}_{10} \mathrm{O}_{28}{ }^{5-}, \mathrm{V}_{10} \mathrm{O}_{28}{ }^{6-}, \mathrm{Mo}_{7} \mathrm{O}_{24}{ }^{6-}$, which decreases mutual solubility with PEG2000-rich phase, leading to lower extraction of vanadium and molybdenum.

\subsubsection{Effect of vanadium concentration}

The effect of the vanadium concentration on co-extraction of vanadium and molybdenum is shown in Fig. 2 . In this figure, we took the mole ratio of vanadium and molybdenum as the abscissa and the extraction rate of vanadium and molybdenum as the ordinate. It can be seen from the figure that when the molar ratio is in the range of $0.3-1.5$, the extraction rate of molybdenum decreases slightly and maintains a higher level, and the extraction rate of vanadium gradually increases from 94.11 to $97.57 \%$ and then decreases slightly as the ratio increases from 1.5 to 3.1 , trace back to the cause is when the aqueous $\mathrm{pH}$ value is below 6.0, and the proportion of vanadium in the heteropolyacid of vanadium and molybdenum exceeds the

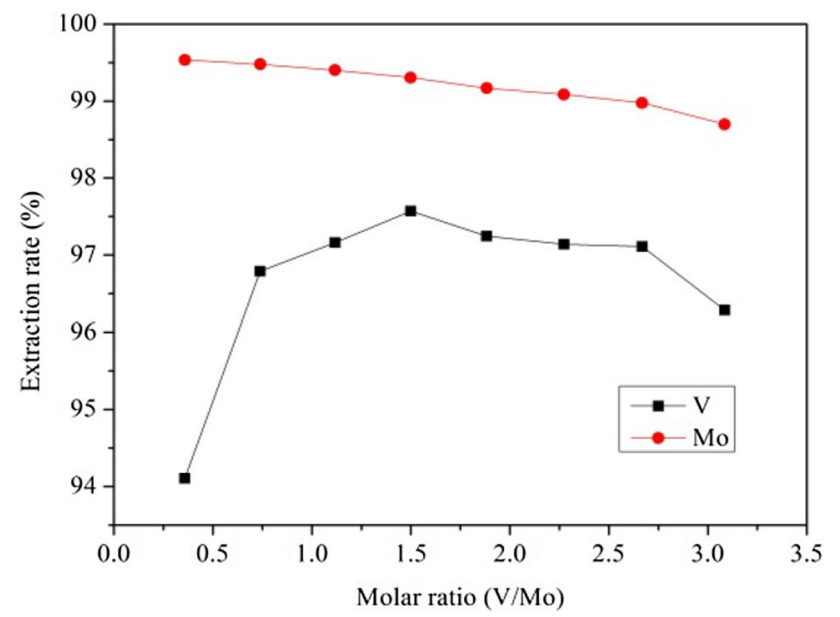

Fig. 2 Effect of vanadium concentration on co-extraction of vanadium and molybdenum (conditions: $\mathrm{pH}: 2.0, \mathrm{~T}: 313.15 \mathrm{~K}, \mathrm{Na}_{2} \mathrm{SO}_{4}$ : 10\%, PEG2000: 20\%) ratio of vanadium in the heteropolyacid [32]. Therefore, when the $\mathrm{pH}$ value is 2.0 , more vanadium and molybdenum continue to form the heteropolyacid as vanadium concentration increases, resulting in an increase in extraction rate of vanadium. However, since the content of molybdenum is a certain amount, when the ratio of vanadium and molybdenum is about 1.5 , the heteropolyacid formed by vanadium and molybdenum reaches "saturation" at this time, and vanadium no longer forms the heteropolyacid as concentration of vanadium continues to increase, leading to excessive vanadium in the aqueous which appears as the form of polyvanadate and resulting in a decrease in extraction rate of vanadium. However, in the scope of this study, the extraction rate of molybdenum decreases slightly with the increase in vanadium concentration and it still remains at a high level of $99.49 \%$.

\subsubsection{Effect of molybdenum concentration}

The effect of molybdenum concentration on co-extraction of vanadium and molybdenum is shown in Fig. 3. It can be observed from the figure that the extraction rate of vanadium rises significantly and the extraction rate of molybdenum is basically unchanged with an increase in molybdenum concentration from 0.1 to $0.8 \%$. With the increase in molybdenum concentration, vanadium and molybdenum easily form heteropolyacid anion in acidic aqueous solution, resulting in an increase in vanadium extraction rate. At the same time, the increase in molybdenum extraction rate is due to the formation of heteropolyacid anion and polymolybdate anion as increasing molybdenum concentration, which is in favor of transferring of molybdenum from salt-rich phase to PEG2000-rich phase

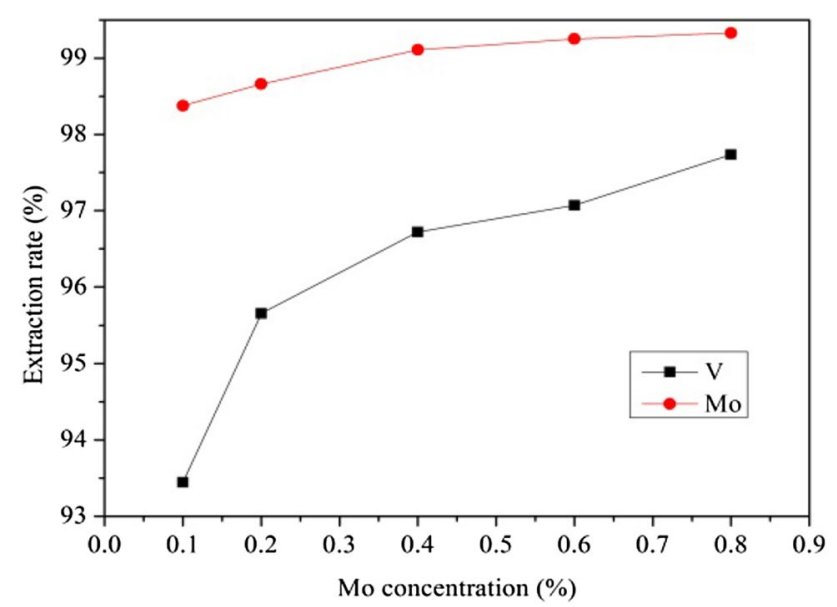

Fig. 3 Effect of molybdenum concentration on co-extraction of vanadium and molybdenum (conditions: $\mathrm{pH}: 2.0, \mathrm{~T}: 313.15 \mathrm{~K}, \mathrm{~V}$ : $0.2 \%, \mathrm{Na}_{2} \mathrm{SO}_{4}: 10 \%$, PEG2000: $20 \%$ ) 
for its relatively high hydrophobic nature and electrostatic attraction between cation and anion.

\subsubsection{Effect of temperature}

The effect of temperature on co-extraction of vanadium and molybdenum is shown in Fig. 4. From the line trend, when temperature increases from 313.15 to $343.5 \mathrm{~K}$, and the extraction rates of vanadium and molybdenum decrease, but the decline is not significant just about one percentage point, which shows that the effect of temperature on extraction performance is not obvious. When the temperature is below $313.15 \mathrm{~K}$, it becomes difficult to form two-phase systems, so the optimal extracting temperature is $313.15 \mathrm{~K}$.

\subsubsection{Extraction isotherm}

The extraction isotherms of molybdenum ( VI) and vanadium (V) with the aqueous two-phase system consisting of $20 \%$ PEG 2000 and $10 \%$ sodium sulfate at $\mathrm{pH}=2.0$ and their McCabe-Thiele diagrams are shown in Fig. $5 \mathrm{a}$ and b, respectively. The operating line was obtained on the bases of countercurrent operation of two phases according to the material conservation equation as follows:

$c_{t, i+1}=\frac{V_{\mathrm{b}}}{V_{\mathrm{t}}} c_{\mathrm{b}, i}+\left(c_{t, 0}-\frac{V_{\mathrm{b}}}{V_{\mathrm{t}}} c_{\mathrm{b}, n}\right)$

where $c_{t^{\prime}+1}$ is the solute concentration in PEG-rich phase left from $i$ theoretical stage, $\mathrm{g} / \mathrm{L} ; \mathrm{C}_{\mathrm{b}^{\prime} i}$ is the solute concentration in salt-rich phase left from $i$ theoretical stage, $\mathrm{g} / \mathrm{L}$; $c_{t \prime} 0$ is the solute concentration in PEG-rich phase added to

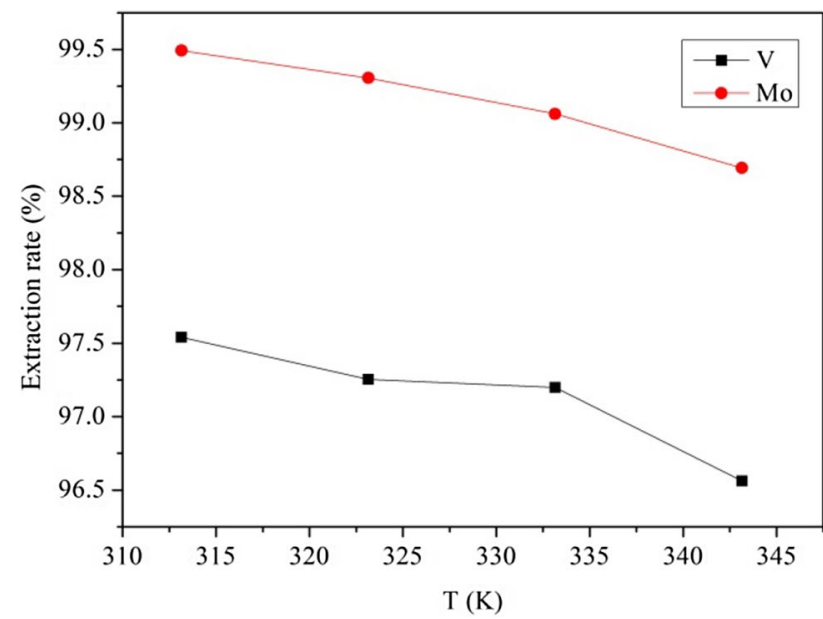

Fig. 4 Effect of temperature on co-extraction of vanadium and molybdenum (conditions: $\mathrm{pH}: 2.0, \mathrm{~V}: 0.2 \%, \mathrm{Mo:} 0.5 \%, \mathrm{Na}_{2} \mathrm{SO}_{4}: 10 \%$, PEG2000: 20\%)
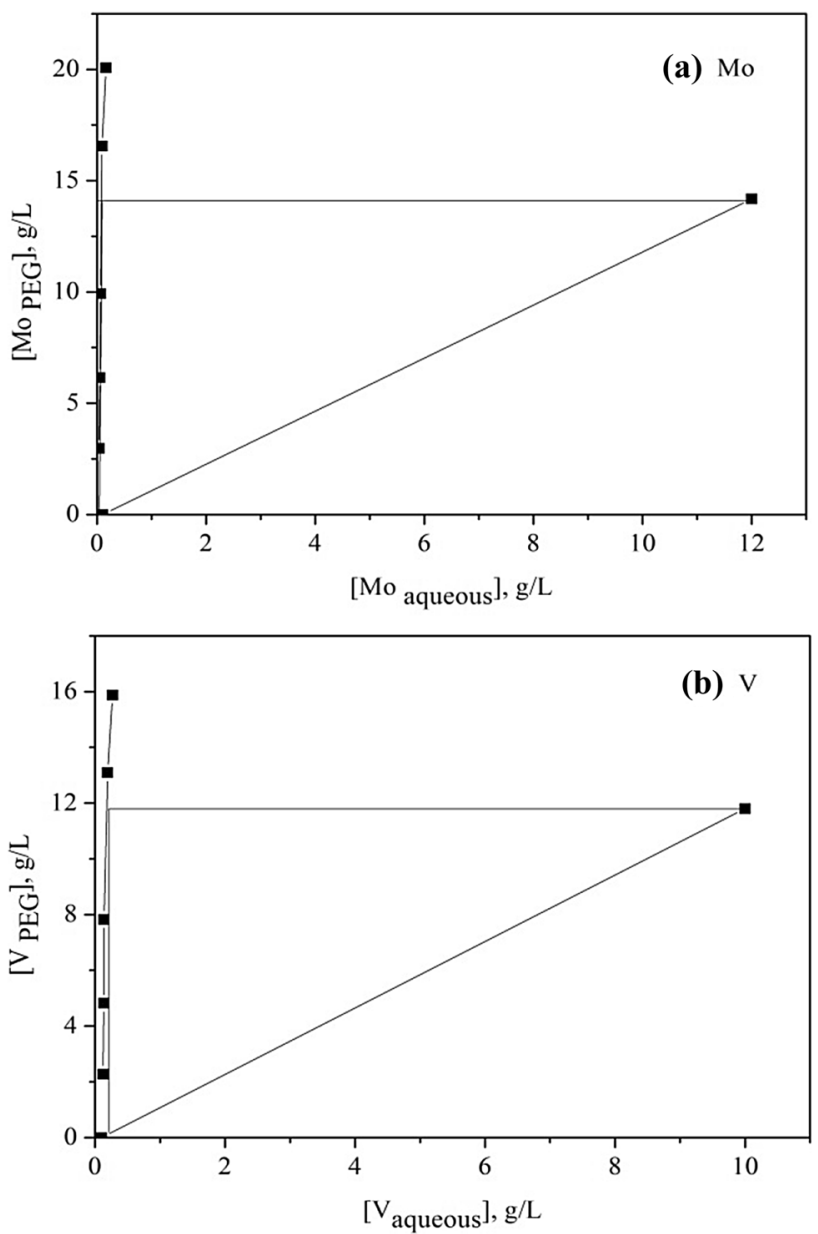

Fig. 5 McCabe-Thiele diagram for determination of the number of stages for complete extraction of molybdenum and vanadium

first theoretical stage, $\mathrm{g} / \mathrm{L} ; C_{\mathrm{b} \prime}$ is the solute concentration in salt-rich phase left from $\mathrm{n}$ theoretical stage, $\mathrm{g} / \mathrm{L}$; and $V_{\mathrm{b}}$ and $V_{\mathrm{t}}$ are the volume of salt-rich phase and PEG-rich phase, respectively, $L$.

It was found from the figures that under the experimental conditions, the possibility to co-extraction of two metals needs only two theoretical extraction stages when molybdenum concentration decreases from initial $12 \mathrm{~g} / \mathrm{L}$ to $0.1 \mathrm{~g} / \mathrm{L}$ and vanadium concentration decreases from initial $10 \mathrm{~g} / \mathrm{L}$ to $0.1 \mathrm{~g} / \mathrm{L}$.

\subsection{Stripping separation of vanadium and molybdenum}

\subsubsection{Effect of $\mathrm{pH}$}

The effect of $\mathrm{pH}$ on stripping rate of vanadium and molybdenum and vanadium precipitation rate is shown in Fig. 6. It can be observed from the figure that when the $\mathrm{pH}$ value changed from 8.0 to 10.0 adjusting by $\mathrm{NH}_{3} \cdot \mathrm{H}_{2} \mathrm{O}$ 


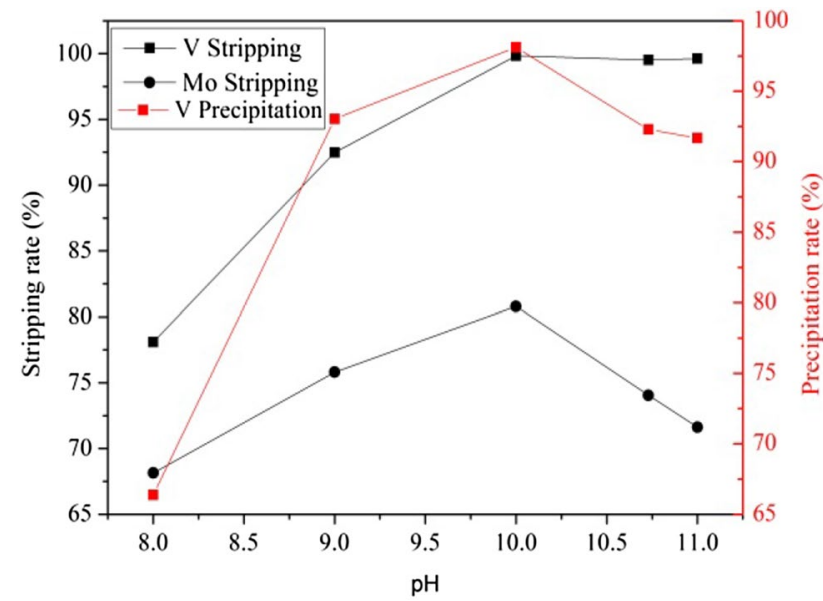

Fig. 6 Effect of aqueous $\mathrm{pH}$ on stripping separation of vanadium and molybdenum (conditions: $\left(\mathrm{NH}_{4}\right)_{2} \mathrm{SO}_{4}: 30 \%, \mathrm{~T}: 343.15 \mathrm{~K}, \mathrm{O} / \mathrm{A}$ : 2/1)

or $\mathrm{H}_{2} \mathrm{SO}_{4}$, the stripping rate and precipitation rate of vanadium increase significantly from 78.10 to $99.84 \%$ and from 66.38 to $98.13 \%$, respectively, and the stripping rate of molybdenum rises from 68.13 to $80.79 \%$. When the $\mathrm{pH}$ value continues to increase to 11.0, the stripping rate of vanadium remains unchanged, and precipitation rate of vanadium decreases, and the stripping rate of molybdenum decreases too. That is due to the different forms of vanadium and molybdenum in solutions of different $\mathrm{pH}$ values. When the $\mathrm{pH}$ is greater than 7.0, vanadium and molybdenum are almost no longer present in the form of polyacid in aqueous solution [33]. During the process of the aqueous solution changing from neutral to alkaline, the morphology of vanadium and molybdenum gradually changed from polyacid to mononuclear anion. When the $\mathrm{pH}$ is greater than 9, vanadium and molybdenum appear only as a mononuclear anion [34].The charge density in the solution increases, the hydrophilicity increases, and the stability of the micelles deteriorates, causing vanadium and molybdenum to be easily dissociated from the inside of the micelle. The metavanadate anion and the ammonium salt form a precipitate of ammonium metavanadate with low solubility, and the molybdenum in the solution is present in the form of ammonium molybdate. In addition, the phase separation is completely in the experimental study $\mathrm{pH}$ range from 8.0 to 10.0. However, when a certain degree is reached, the precipitation rate of vanadium decreases due to the small amount of ammonium metavanadate dissolved in excess ammonia water. Therefore, there is a good separation effect on vanadium and molybdenum when the stripping $\mathrm{pH}$ is 10.0 .

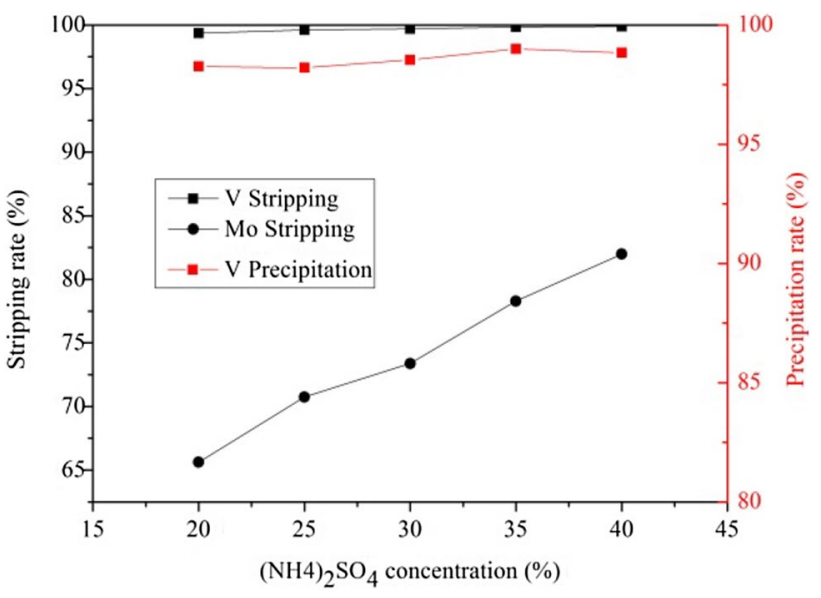

Fig. 7 Effect of $\left(\mathrm{NH}_{4}\right)_{2} \mathrm{SO}_{4}$ concentration on stripping separation of vanadium $(\mathrm{V})$ and molybdenum $(\mathrm{VI})$ (conditions: $\mathrm{pH}$ : 10.0, $\mathrm{T}$ : $343.15 \mathrm{~K}, \mathrm{O} / \mathrm{A}: 2 / 1)$

\subsubsection{Effect of $\left(\mathrm{NH}_{4}\right)_{2} \mathrm{SO}_{4}$ concentration}

All kinds of stripping reagents, such as ammonium carbonate and ammonium hydroxide, ammonium sulfate and ammonium hydroxide, were tested to determine their stripping performance on vanadium and molybdenum, it was found that ammonium sulfate and ammonium hydroxide was advantageous as stripping reagents, and their the stripping effect was the best for remarkable salting-out effect, non-emulsification phenomenon, clearly split-phase, limpid salt phase. The effect of $\left(\mathrm{NH}_{4}\right)_{2} \mathrm{SO}_{4}$ concentration varying from 20 to $40 \%$ on stripping rate of vanadium and molybdenum and vanadium precipitation rate is shown in Fig. 7. It can be seen from the figure that the stripping rate of molybdenum is significantly affected by $\left(\mathrm{NH}_{4}\right)_{2} \mathrm{SO}_{4}$ concentration. As $\left(\mathrm{NH}_{4}\right)_{2} \mathrm{SO}_{4}$ concentration increases from 20 to $40 \%$, the stripping rate of molybdenum increases from 65.62 to $82.00 \%$. This is due to the fact that after the addition of $\left(\mathrm{NH}_{4}\right)_{2} \mathrm{SO}_{4}$, heteropolyacid anions of vanadium and molybdenum are broke and ammonium molybdate are formed in alkaline condition, in the meanwhile, the salting-out effect of $\left(\mathrm{NH}_{4}\right)_{2} \mathrm{SO}_{4}$ makes dehydration in PEG2000-rich phase, leading to the increase in hydrophobic nature in PEG2000-rich phase, which results in decreasing mutual solubility between ammonium molybdate and hydrophobic PEG2000-rich phase. The stripping rate and precipitation rate of vanadium were not significantly affected by $\left(\mathrm{NH}_{4}\right)_{2} \mathrm{SO}_{4}$, and with the increase in $\left(\mathrm{NH}_{4}\right)_{2} \mathrm{SO}_{4}$ concentration, the stripping rate and precipitation rate of vanadium remained at a high level of $99.88 \%$ and $98.84 \%$, respectively. It indicates that the stripping rate of molybdenum is mainly affected by the salting out of $\left(\mathrm{NH}_{4}\right)_{2} \mathrm{SO}_{4}$, and then, the stripping rate and precipitation rate of vanadium is less affected by it. 


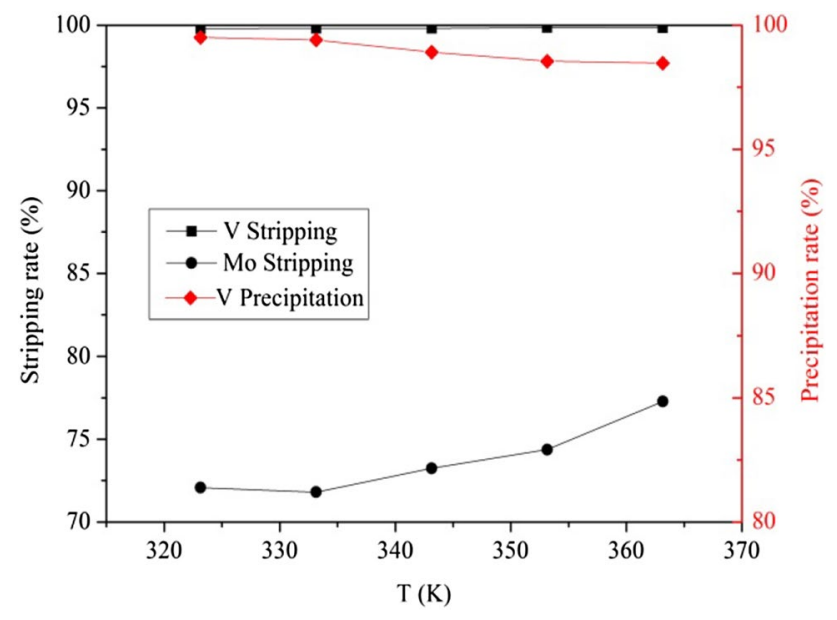

Fig. 8 Effect of temperature on stripping separation of vanadium and molybdenum (conditions: $\mathrm{pH}: 10.0,\left(\mathrm{NH}_{4}\right)_{2} \mathrm{SO}_{4}: 35 \%, \mathrm{O} / \mathrm{A:}: 2 / 1$ )

\subsubsection{Effect of temperature}

The effect of temperature varying from 323.15 to $363.15 \mathrm{~K}$ on stripping rate of vanadium and molybdenum and vanadium precipitation rate is shown in Fig. 8. It can be seen from the figure that with the gradual increase in temperature from 323.15 to $363.15 \mathrm{~K}$, the stripping rate of molybdenum increases from 72.07 to $77.28 \%$, indicating that the stripping rate of molybdenum is greatly affected by temperature. That is because PEG2000 in aqueous solution is achieved by hydrogen bonding of hydrophilic molecules in the PEG2000 with water molecules, and the formation of hydrogen bonds is an exothermic process, so as the temperature rising, the binding force of the hydrogen bonds will be weakened or even disappeared, and dehydration will occur, which will facilitate the separation of molybdenum from the micellar phase, leading to an increase in the stripping rate. The stripping rate and precipitation rate of vanadium are less affected by temperature and are basically maintained at a high level of over $99.85 \%$ and $98.47 \%$, respectively.

\subsubsection{Effect of phase ratio}

The effect of phase ratio varying from 1.0 to 4.0 (mass ratio of PEG2000-rich phase and ammonium sulfate aqueous phase) on stripping rate of vanadium and molybdenum and vanadium precipitation rate is shown in Fig. 9. It can be seen from the figure that as the phase ratio value increases from 1.0 to 4.0 , and the stripping rate of molybdenum decreases significantly from 81.83 to $60.52 \%$, while the stripping rate and precipitation rate of vanadium remain high level above $99.63 \%$ and $98.71 \%$, respectively, which indicates that the small phase ratio value facilitates

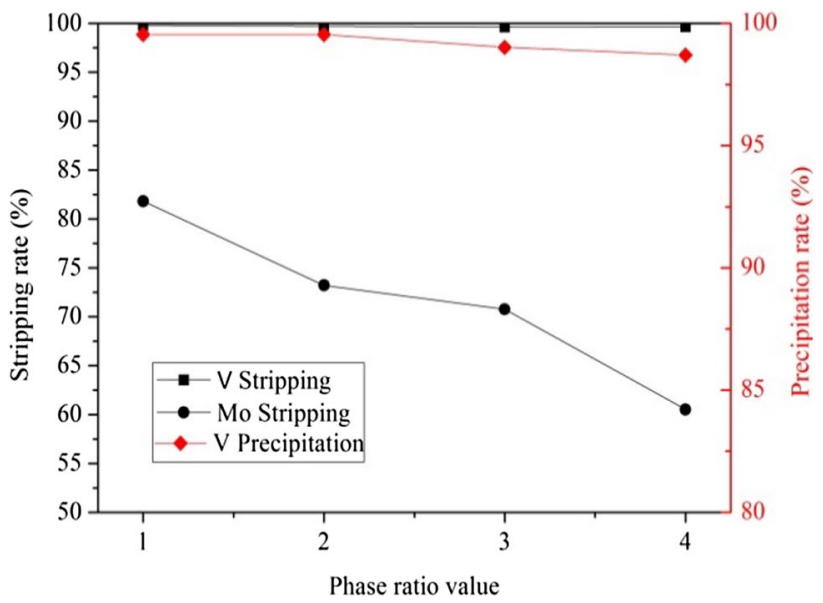

Fig. 9 Effect of phase ratio value on stripping separation of vanadium and molybdenum (conditions: $\mathrm{pH}$ : $10.0,\left(\mathrm{NH}_{4}\right)_{2} \mathrm{SO}_{4}: 30 \%$, T: $343.15 \mathrm{~K}$ )

the transfer of vanadium and molybdenum from PEG2000rich phase to stripping aqueous phase.

After a single-stage contact, the stripping rate and precipitation rate of vanadium(V) reach $99.88 \%$ and $98.84 \%$, respectively, and the stripping rate of molybdenum(VI) reaches $81.99 \%$. The stripping efficiency of vanadium and molybdenum is listed in Table 1 under the optimal stripping conditions in a single-stage contact. There is only $0.74 \%$ molybdenum in the product of vanadium pentoxide and $0.578 \%$ vanadium in the product of molybdenum trioxide, showing that the separation of vanadium and molybdenum was realized efficiently.

In order to further prove the separated products, the separated products were roasted in a constant temperature muffle furnace at $500^{\circ} \mathrm{C}$ for $2 \mathrm{~h}$ and analyzed by XRD. The analysis results are shown in Fig. 10a and b. Compared with the standard cards, it can be proved that the products are $\mathrm{V}_{2} \mathrm{O}_{5}$ and $\mathrm{MoO}_{3}$, respectively.

\section{Conclusions}

In the absence of any extractant, a method for the efficiently co-extraction and selective stripping separation of vanadium(V) and molybdenum(VI) by aqueous two-phase systems consisting of PEG $2000+$ sodium sulfate $+\mathrm{H}_{2} \mathrm{O}$ and ammonium sulfate + loaded PEG2000 phase was proposed in this study. The main conclusions are as follows: The coextraction rate of vanadium and molybdenum is highest at $\mathrm{pH}=2.0$, showing that these heteropolyacid anions have relative high hydrophobicity and increase mutual solubility with PEG2000-rich phase having hydrophobic environment, in the meanwhile, the electrostatic attraction generated between heteropolyacid anion and quasi-cation of 
Table 1 Stripping efficiency of both metals in a single-stage contact

\begin{tabular}{|c|c|c|c|c|c|c|}
\hline \multirow[t]{2}{*}{ Samples } & \multicolumn{2}{|c|}{ Concentration (mg/L) } & \multicolumn{2}{|c|}{ Stripping (\%) } & \multicolumn{2}{|c|}{$\begin{array}{l}\text { Precipitation rate } \\
(\%)\end{array}$} \\
\hline & Mo & V & Mo & V & Mo & V \\
\hline PEG2000 phase & 5345 & 8.125 & - & - & - & - \\
\hline Stripping liquor & 25,825 & 115 & 81.99 & 99.88 & - & 98.84 \\
\hline
\end{tabular}
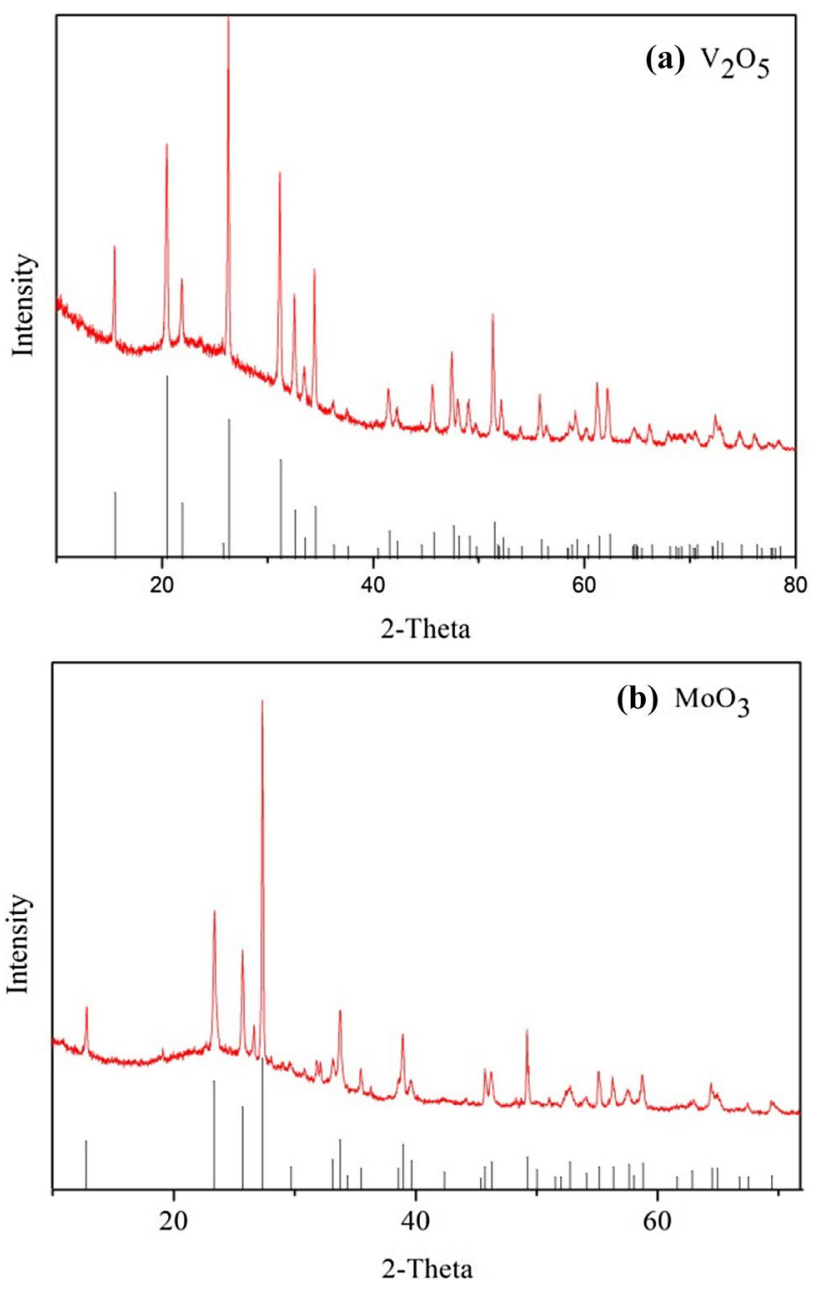

Fig. 10 XRD diagrams of the roasted products, $\mathbf{a ~} \mathrm{V}_{2} \mathrm{O}_{5}, \mathbf{b} \mathrm{MoO}_{3}$

$\mathrm{C}-\mathrm{O}-\mathrm{C}$ or $-\mathrm{OH}$ protonated in PEG2000 molecule promotes the extraction rate of heteropolyacid anion. What's more, the increase in the heteropolyacid content caused by the increase in the concentration of molybdenum can lead to the increase in the co-extraction rate of vanadium and molybdenum. Increasing extraction temperature is not in favor of the co-extraction of vanadium and molybdenum Under the appropriate conditions of maintaining the $\mathrm{pH}$ value of the aqueous solution at 2.0, the molar ratio of vanadium to molybdenum is about 1.5 , the concentration of PEG 2000 at $20 \%$ and $313.15 \mathrm{~K}, 97.54 \%$ of vanadium and $99.49 \%$ of molybdenum can be extracted into the
PEG2000-rich phase. Two theoretical extraction stages are demanded when molybdenum concentration decreases from initial $12 \mathrm{~g} / \mathrm{L}$ to $0.1 \mathrm{~g} / \mathrm{L}$ and vanadium concentration decreases from initial $10 \mathrm{~g} / \mathrm{L}$ to $0.1 \mathrm{~g} / \mathrm{L}$ by McCabe-Thiele method. For selective stripping of vanadium and molybdenum, when the $\mathrm{pH}$ of the system varies from 8.0 to 10.0 , the charge density in the solution increases, the hydrophilicity increases, and the stability of the micelles deteriorates, causing vanadium and molybdenum to be easily dissociated from the micelle phase. Under alkaline conditions and adding ammonium sulfate, the heteropolyacid anion of vanadium and molybdenum was broken and ammonium molybdate and ammonium metavanadate were formed, resulting in the removal of vanadium and molybdenum from PEG2000-rich micelle phase because of increasing hydrophilic nature of both metals. However, the stripping rate and precipitation rate of vanadium are not significantly changed by the above factors. By using a $40 \%\left(\mathrm{NH}_{4}\right)_{2} \mathrm{SO}_{4}$ solution, above $99.88 \%$ of vanadium and $81.99 \%$ of molybdenum can be stripped and $98.84 \%$ of vanadium can be precipitated from surfactant loaded with vanadium and molybdenum in one step under conditions of $O / A=2: 1$, stripping temperature $343.15 \mathrm{~K}$ and the $\mathrm{pH}$ of aqueous solution 10.0. An efficient separation of vanadium and molybdenum in aqueous solution is achieved. We hope that this new separation method can be used as an alternative scheme for industrial production in the future.

Acknowledgements The authors wish to thank natural science foundation of Hebei Province, China, for their financial support (E2019203538).

\section{Compliance with ethical standards}

Conflict of interest The author(s) declare that they have no conflict of interests

\section{References}

1. Marafi M, Stanislaus A (2008) Spent hydroprocessing catalyst management: a review: part II. Advances in metal recovery and safe disposal methods. Resour Conserv Recycl 53:1-26

2. Akcil A, Vegliò F, Ferella F, Okudan MD, Tuncuk A (2015) A review of metal recovery from spent petroleum catalysts and ash. Waste Manage 45:420-433 
3. Furimsky E (1996) Spent refinery catalysts: environment, safety and utilization. Catal Today 30:223-286

4. Chen Y, Feng QM, Shao YH, Zhang GF, Ou LM, Lu YP (2006) Investigations on the extraction of molybdenum and vanadium from ammonia leaching residue of spent catalyst. Int J Miner Process 79:42-48

5. Kim HI, Lee KW, Mishra D, Yi KM, Hong JH, Jun MK, Park HK (2014) Separation and recovery of vanadium from leached solution of spent residue hydrodesulfurization (RHDS) catalyst using solvent extraction. J Ind Eng Chem 20:4457-4462

6. Biswas RK, Wakihara M, Taniguchi M (1985) Recovery of vanadium and molybdenum from heavy oil desulphurization waste catalyst. Hydrometallurgy 14:219-230

7. Banda R, Nguyen TH, Sohn SH, Lee MS (2013) Recovery of valuable metals and regeneration of acid from the leaching solution of spent HDS catalysts by solvent extraction. Hydrometallurgy 133:161-167

8. Park KH, Mohapatra D, Reddy BR (2006) Selective recovery of molybdenum from spent HDS catalyst using oxidative soda ash leach/carbon adsorption method. J Hazard Mater 132:311-316

9. Sahu KK, Agrawal A, Mishra D (2013) Hazardous waste to materials: recovery of molybdenum and vanadium from acidic leach liquor of spent hydroprocessing catalyst using alamine 308 . J Environ Manage 125:68-73

10. Nguyen TH, Lee MS (2014) Recovery of molybdenum and vanadium with high purity from sulfuric acid leach solution of spent hydrodesulfurization catalysts by ion exchange. Hydrometallurgy 147-148:142-147

11. Nguyen TH, Lee MS (2013) Separation of molybdenum and vanadium from acid solutions by ion exchange. Hydrometallurgy 136:65-70

12. Zhu ZW, Tulpatowicz K, Pranolo Y, Cheng CY (2015) Solvent extraction of molybdenum and vanadium from sulphate solutions with Cyphos IL 101. Hydrometallurgy 154:72-77

13. Truong HT, Nguyen TH, Lee MS (2017) Separation of molybdenum(VI), rhenium(VII), tungsten( $\mathrm{VI})$, and vanadium(V) by solvent extraction. Hydrometallurgy 171:298-305

14. Yaftian MR, Almeida MIGS, Cattrall RW, Kolev SD (2018) Selective extraction of vanadium (V) from sulfate solutions into a polymer inclusion membrane composed of poly(vinylidenefluoride-cohexafluoropropylene) and Cyphos IL 101. J Membr Sci 545:57-65

15. Chen Y, Feng QM, Zhang GF, Ou L, Lu Y (2007) Study on the recycling of valuable metals in spent $\mathrm{Al}_{2} \mathrm{O}_{3}$-based catalyst. Miner Metall Process 24:30-34

16. Kim HI, Lee KW, Mishra D, Yi KM, Hong JH, Jun MK, Park HK (2015) Separation of molybdenum and vanadium from oxalate leached solution of spent residue hydrodesulfurization (RHDS) catalyst by liquid-liquid extraction using amine extractant. J Ind Eng Chem 21:1265-1269

17. Li XB, Wei C, Wu J, Li MT, Deng ZG, Li CX, Xu HS (2012) Co-extraction and selective stripping of vanadium (IV) and molybdenum (VI) from sulphuric acid solution using 2-ethylhexyl phosphonic acid mono-2-ethylhexyl ester. Sep Purif Technol 86:64-69

18. Zhang PW, Inoue K, Yoshizuka K, Tsuyama H (1996) Extraction and selective stripping of molybdenum(VI) and vanadium(IV) from sulfuric acid solution containing aluminum(III), cobalt(II), nickel(II) and iron(III) by LIX 63 in Exxsol D80. Hydrometallurgy 41:45-53

19. Zhang Y, Zhang TA, Dreisinger D, Zhou WH, Xie F, Lv GZ, Zhang WG (2018) Chelating extraction of vanadium (V) from low $\mathrm{pH}$ sulfuric acid solution by mextral $973 \mathrm{H}$. Sep Purif Technol 190:123-135
20. Shibukawa M, Ichikawa R, Baba T, Sakamoto R, Saito S, Oguma K (2008) Separation selectivity of aqueous polyethylene glycolbased separation systems: DSC, LC and aqueous two-phase extraction studies. Polymer 49:4168-4173

21. Lee KW, How CW, Chen L, Chen PT, Lan JCW, Ng HS (2019) Integrated extractive disruption of Gordonia terrae cells with direct recovery of carotenoids using alcohol/salt aqueous biphasic system. Sep Purif Technol 223:107-112

22. Nascimento CO, Soares PAG, Porto TS, Costa RMPB, Lima CDA, Fiho JLDL, Coelho LCBB, Correia MTDS, Cunha MDGC, Porto ALF (2013) Aqueous two-phase systems: new strategies for separation and purification of lectin from crude extract of Cratylia mollis, seeds. Sep Purif Technol 116:154-161

23. Antov MG (2004) Partitioning of pectinase produced by Polyporus squamosus in aqueous two-phase system polyethylene glycol 4000/crude dextran at different initial $\mathrm{pH}$ values. Carbohyd Polym 56:295-300

24. Souza RL, Farias FO, Mafra MR, Soares CMF, Passos H, Coutinho JAP (2019) Acetonitrile as adjuvant to tune polyethylene gly$\mathrm{col}+\mathrm{K}_{3} \mathrm{PO}_{4}$ aqueous two-phase systems and its effect on phenolic compounds partition. Sep Purif Technol 223:41-48

25. Hamta A, Dehghani MR (2017) Application of polyethylene glycol based aqueous two-phase systems for extraction of heavy metals. J Mol Liq 231:20-24

26. Santos LH, Carvalho PLG, Rodrigues GD, Mansur MB (2015) Selective removal of calcium from sulfate solutions containing magnesium and nickel using aqueous two phase systems (ATPS). Hydrometallurgy 156:259-263

27. Paik SP, Sen K (2018) Extraction of Bi(III) subsalicylate in micellar aggregation and its sustained releases using an aqueous biphasic system. J Mol Liq 249:188-192

28. Wang P, Zhang F, Li P, Sun TT, Pan YJ, Zhang YQ (2018) Partitioning performance of molybdenum in poly (ethylene glycol) + sodium sulfate + water aqueous two-phase systems. J Mol Liq 260:180-185

29. Zhang YQ, Sun TC, Lu TQ, Yan CH (2016) Extraction and separation of tungsten $(\mathrm{VI})$ from aqueous media with Triton X-100-ammonium sulfate-water aqueous two-phase system without any extractant. J Chromatogr A 1474:40-46

30. Zhang YQ (2019) Study on partitioning performance of vanadium, molybdenum and tungsten in nonionic surfactant aqueous two-phase systems, Doctoral Dissertation, University of Science and Technology of Beijing

31. Durán A, Claros M, Jimenez YP (2018) Molybdate ion partition in the aqueous two-phase system formed by $\mathrm{CuSO}_{4}+\mathrm{PEG}$ $4000+\mathrm{H}_{2} \mathrm{O}$ at different $\mathrm{pH}$ and temperatures. J Mol Liq 249:562-572

32. Liao $\mathrm{YL}$, $\mathrm{Li}$ JT (2016) $\mathrm{Mo}(\mathrm{VI})-\mathrm{V}(\mathrm{V})-\mathrm{H}_{2} \mathrm{O}$ thermodynamic analysis of the system. Powder Metall Mater Sci Eng 21:678-684

33. Olazabal MA, Orive MM, Fernandez LA, Madariaga JM (1992) Selective extraction of vanadium( $(V)$ from solutions containing molybdenum $(\mathrm{VI})$ by ammonium salts dissolved in toluene. Solvent Extr lon Exch 10:623-635

34. Bal KE, Cote G, Lallam A, Bal Y (2004) Characterization of the solid third phases that precipitate from the organic solutions of Aliquat 336 after extraction of molybdenum(VI) and vanadium(V). Hydrometallurgy 75:123-134

Publisher's Note Springer Nature remains neutral with regard to jurisdictional claims in published maps and institutional affiliations. 